\title{
MODELAGEM MATEMÁTICA DE COPOLIMERIZAÇÃO EM EMULSÃO DE ACRILATO DE BUTILA E ESTIRENO PARA DETERMINAÇÃO DA DISTRIBUIÇÃO DE TAMANHO DE PARTÍ́CULAS.
}

\author{
R. V. PEREIRA ${ }^{1}$ e R. GIUDICI ${ }^{1}$ \\ ${ }^{1}$ Universidade de São Paulo (USP), Escola Politécnica, Departamento de Engenharia Química \\ E-mail para contato: rvallejo@usp.br
}

\begin{abstract}
RESUMO - A construção de um modelo matemático da reação de copolimerização em emulsão de acrilato de butila e estireno em reator semi-batelada e isotérmico apresentou bons resultados quando comparado aos experimentos. O modelo contemplou a solução do balanço populacional, tanto para a distribuição de tamanho de partículas quando para a distribuição média de radicais por partícula. O problema descrito foi resolvido através da solução numérica de um conjunto de equações algébrico-diferenciais. O modelo foi validado com dados experimentais de concentração de monômeros na fase aquosa, diâmetro médio de partículas, número de partículas por litro de emulsão, número médio de radicais por partícula e a distribuição de tamanho de partículas ao longo do tempo.
\end{abstract}

\section{INTRODUÇÃO}

O processo de polimerização em emulsão é usado para a produção de uma grande variedade de produtos incluindo adesivos, tintas, colas, aditivos para papel, aditivos para têxteis, aditivos para materiais de construção, modificadores de impactos para plásticos e em sistema de liberação de fármacos. A variedade de produtos que podem ser produzidas expandiu com o desenvolvimento de técnicas de polimerização em miniemulsão e polimerização radicalar controlada (reversivelmente desativada) em meio disperso (ASUA, 2003).

A polimerização em emulsão é reconhecida como um processo heterogêneo complexo, envolvendo transporte de monômeros, radicais livres e de outras espécies entre as fases aquosa e orgânica. Comparando com outros processos heterogêneos de polimerização, como polimerização em suspensão e precipitação, a polimerização em emulsão apresenta-se como o processo mais complicado. A taxa de polimerização na fase orgânica não é controlada somente pela partição de monômero, mas também por outros fenômenos como nucleação de partículas, absorção e dessorção de radicais. A complexidade do sistema aumenta devido às interações que existem entre as fases e os mecanismos de difusão e polimerização dos componentes envolvidos. Ainda, a estabilidade das partículas é afetada pelo tipo e quantidade e força iônica do surfactante no meio disperso (GAO e PENLIDIS, 2002; ARAÚJO e GIUDICI, 2003; ARAÚJO e GIUDICI, 2004).

A modelagem matemática e o seu papel na engenharia são pontos de constante debate, 


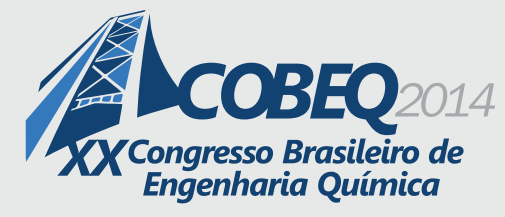

principalmente quando os modelos são implementados em ambientes industriais. Entretanto, os modelos nos auxiliam no entendimento dos fenômenos envolvidos nos processos de interesse e nos ajudam no direcionamento da experimentação. Eles funcionam como uma reserva de conhecimento e podem nos auxiliar no entendimento de interações que seriam dificilmente previstas somente com experimentos, principalmente quando muitos fatores estão envolvidos. Os modelos são importantes para projeto, estimação de parâmetros, análises de sensibilidade, simulação, controle e otimização de processos e ainda evitam o desperdício de custos com experimentos (DUBÉ et al., 1997).

Diversos modelos matemáticos para processos de polimerização em emulsão têm sido desenvolvidos na literatura, com diferentes graus de complexidade. O presente trabalho objetivou desenvolver um modelo capaz de prever a distribuição de tamanhos das partículas, que é uma importante variável de qualidade da emulsão produzida, influindo em propriedades reológicas da emulsão polimérica.

\section{MODELAGEM MATEMÁTICA}

\subsection{Reações Químicas}

Na Tabela 1 são apresentadas as reações químicas do mecanismo de polimerização radicalar consideradas no desenvolvimento do modelo, com a indicação da fase em que estas reações ocorrem. Os fenômenos relacionados à transferência entre fases também são considerados descritos através da notação de reações químicas conforme a Tabela 2.

Tabela 1 - Reações químicas envolvidas no processo de polimerização em emulsão

\begin{tabular}{l|l|l}
\hline Fenômeno & Reação & Fase \\
\hline Decomposição do Iniciador & $I \stackrel{k_{d}}{\longrightarrow} 2 \dot{R}_{i n}$ & Aquosa \\
Iniciação & $\dot{R}_{i n}+M_{j} \stackrel{k_{i}}{\longrightarrow} \dot{R}_{1, j}$ & Aquosa \\
Propagação & $\dot{R}_{m, i}+M_{j} \stackrel{k_{p}}{\longrightarrow} \dot{R}_{m+1, j}$ & Aquosa/Polímero \\
Transferência para Monômero & $\dot{R}_{m, i}+M_{j} \stackrel{k_{f m}}{\longrightarrow} D_{m}+\dot{R}_{1, j}$ & Aquosa/Polímero \\
Transferência para Polímero & $\dot{R}_{m, i}+D_{r} \stackrel{k_{f p}}{\longrightarrow} D_{m}+\dot{R}_{r, j}$ & Polímero \\
Transferência para CTA & $\dot{R}_{m, i}+A \stackrel{k_{f A}}{\longrightarrow} D_{m}+\dot{A}$ & Polímero \\
Terminação de CTA & $\dot{A}+M_{j} \stackrel{k_{t A}}{\longrightarrow} \dot{R}_{1, j}$ & Polímero \\
Terminação Combinação & $\dot{R}_{m, i}+\dot{R}_{r, j} \stackrel{k_{t c}}{\longrightarrow} D_{m+r}$ & Aquosa/Polímero \\
Terminação Desproporcionamento & $\dot{R}_{m, i}+\dot{R}_{r, j} \stackrel{k_{t d}}{\longrightarrow} D_{m}+D_{r}$ & Aquosa/Polímero \\
\hline
\end{tabular}

Onde: $I=$ iniciador, $\dot{R}_{i n}=$ radicais provenientes da decomposição de iniciadores, $M_{j}=$ monômero de tipo $j, \dot{R}_{m, j}=$ radicais de tamanho $m$ e terminação do tipo $j, D_{m}=$ polímeros mortos de tamanho $m, A=$ agente de transferência de cadeia (CTA), $\dot{A}=$ radical proveniente do CTA. 
Tabela 2 - Fenômenos de transferência de radicais entre a fase aquosa e fase partícula

\begin{tabular}{l|l|l}
\hline Fenômeno & Reação & Fase \\
\hline Nucleação Heterogênea & {$\left[\dot{R}_{m, i}\right]_{a q}+$ Mic. $\longrightarrow\left[\dot{R}_{m, i}\right]_{a q}+$ Part. } & $\begin{array}{c}\text { Aquosa } \rightarrow \text { Partícula } \\
(z<m<j c r i t)\end{array}$ \\
Nucleação Homogênea & {$\left[\dot{R}_{j c r i t-1, i}\right]_{a q}+M_{j} \longrightarrow\left[\dot{R}_{j c r i t, j}\right]_{p}+$ Part. } & $\begin{array}{c}\text { Aquosa } \rightarrow \text { Partícula } \\
(z<m<j c r i t)\end{array}$ \\
Captura de Radicais & {$\left[\dot{R}_{m, i}\right]_{a q}+$ Part. $\longrightarrow\left[\dot{R}_{m, i}\right]_{p}+$ Part. } & Aquosa $\rightarrow$ Partícula \\
Dessorção & {$\left[\dot{R}_{1, j}\right]_{p} \longrightarrow\left[\dot{R}_{1, j}\right]_{a q}$} & Partícula $\rightarrow$ Aquosa \\
\hline
\end{tabular}

Onde: Mic. $=$ micela, Part. $=$ partícula de polímero, $j_{c r i t} \cdot=$ índice que identifica se oligômero sofrerá precipitação gerando uma partícula por nucleação homogênea, $z$ = índice que determina se o tamanho oligômero na fase aquosa tem hidrofobicidade suficiente para poder ser capturado por micelas ou partículas.

\subsection{Balanço Populacional}

O balanço populacional para o tamanho de partículas na polimerização em emulsão pode ser descrito, considerando os termos de nucleação, crescimento e coagulação. É importante ressaltar que na polimerização em emulsão não há efeito de quebra das partículas. A equação 1 representa o balanço populacional para polimerização em emulsão (MARINANGELO et al., 2011).

$$
\frac{\partial F(r, t)}{\partial t}+\frac{\partial}{\partial r}\left(F(r, t) \cdot \frac{d r}{d t}\right)=\Re_{N u c} \cdot \delta\left(r-r_{n u c}\right)
$$

A função $\delta\left(r-r_{\text {nuc }}\right)=1$ se $r=r_{\text {nuc }}$, senão $\delta\left(r-r_{\text {nuc }}\right)=0$. Pode-se calcular a taxa de crescimento das partículas $(d r / d t)$ através da equação 2 .

$$
\frac{d r}{d t}=\frac{1}{4 \pi \cdot r^{2} \cdot \rho_{p o l}} \sum_{i}^{A, B} \sum_{j}^{A, B} k_{p i j} \cdot p_{i} \cdot \frac{\tilde{n}}{N_{A}} \cdot\left[M_{j}\right]_{p} \cdot M M_{j}
$$

A taxa de nucleação pode ser definida como a soma das contribuições dos mecanismos de nucleação micelar e nucleação homogênea, na forma:

$$
\begin{aligned}
& \mathfrak{R}_{\text {Nuc }}=\mathfrak{R}_{\text {Mic }}+\mathfrak{R}_{\text {Homog }} \\
& \mathfrak{R}_{\text {Mic }}=\sum_{m=z}^{j c r i t-1} \sum_{i}^{A, B} k_{\text {emic }, i} \cdot p_{a q, i} \cdot\left[\dot{R}_{m}\right]_{a q} \cdot N_{\text {mic }} \quad z<m<j_{\text {crit }} \\
& \mathfrak{R}_{\text {Homog }}=\left(\sum_{i}^{A, B} \sum_{j}^{A, B} k_{p i j} \cdot p_{a q, i} \cdot\left[M_{j}\right]_{a q}\right) \cdot\left[\dot{R}_{j c r i t-1}\right]_{a q} \cdot V_{a q}
\end{aligned}
$$




\subsection{Número médio de radicais nas partículas}

O número médio de radicais por partícula pode também ser modelado através de um balanço populacional, considerando as taxas de entrada, dessorção e terminação. A coagulação não é considerada.

$$
\frac{d \tilde{n}(r, t)}{d t}=\mathfrak{R}_{E n t}^{\prime}(r)-\mathfrak{R}_{D e s}^{\prime}(r)-2 \cdot \mathfrak{R}_{T e r}^{\prime}(r)
$$

\subsection{Radicais na fase aquosa}

Considera-se a iniciação através de reações tipo redox, o cálculo de radicais na fase aquosa é feito através da hipótese do estado pseudo-estacionário. O radical de tamanho $j_{\text {crit }}$. define o tamanho que os radicais passam a ser insolúveis na fase aquosa e geram uma nova partícula de polímero. Este tamanho crítico depende da solubilidade do monômero na fase aquosa. O radical de tamanho $z$ é o menor radical que pode entrar em uma micela ou em uma partícula de polímero (ARAÚJO e GIUDICI, 2003; ARAÚJO e GIUDICI, 2004).

$$
\begin{aligned}
& {\left[\dot{R}_{1}\right]_{a q}=\frac{2 \cdot f \cdot k_{d} \cdot[I]+\frac{1}{V_{R}} \cdot\left[\int_{r_{n u c}}^{r_{\max }} \tilde{n}(r, t) \cdot F(r, t) \cdot d r\right] \cdot \sum_{j}^{A, B} k_{f m j} \cdot\left[M_{j}\right]_{p}}{\sum_{j}^{A, B} k_{p j, a q} \cdot\left[M_{j}\right]_{a q}+\bar{k}_{t, a q} \cdot\left[\dot{R}_{T}\right]_{a q}}} \\
& {\left[\dot{R}_{T}\right]_{a q}=\left[\dot{R}_{1}\right]_{a q} \cdot\left[1+\sum_{n=2}^{z-1} \alpha^{n-1}+\alpha^{z-2} \cdot \sum_{n=z}^{j_{c r i t-1}} \beta^{n-(z-1)}\right]}
\end{aligned}
$$

Os parâmetros $\alpha$ e $\beta$ pode ser calculados através das concentrações das diversas espécies na fase aquosa, pelas constantes de reação, pelo número de micelas e partículas no reator.

\subsection{Partição de Monômeros}

Adota-se a abordagem em que a concentração de cada componente em cada uma das fases está em equilíbrio termodinâmico e as suas concentrações são calculadas pelos coeficientes de partição.

\subsection{Partição de Surfactante}

O emulsificante pode estar presente dissolvido na fase aquosa livre, na forma de micelas, adsorvido na superfície das partículas de polímero ou das gotas de monômero. Entretanto, a área total das gotas de monômero é muito menor que área total das partículas e assim se negligencia a quantidade de emulsificante adsorvido nas gotas de monômero (ARAÚJO e GIUDICI, 2003). A partir destas premissas, calcula-se o número de micelas através da concentração de emulsificante e da concentração micelar crítica. 


\subsection{Balanço de massa}

O balanço de massa do processo de polimerização em emulsão pode ser descrito considerando cada um dos componentes. As reações químicas apresentadas na tabela 1 são a fonte principal para a construção do sistema de equações diferenciais apresentadas nos tópicos abaixo.

\section{RESULTADOS E DISCUSSÃO}

Uma amostra dos resultados do modelo é apresentada a seguir, comparando as curvas simuladas com dados experimentais no grupo de pesquisa, reportados no trabalho de MARINANGELO (2011). Os dados se referem a um experimento iniciado em processo semibatelada, com alimentação de pré-emulsão de monômeros (estireno e acrilado de butila) e de solução aquosa de iniciador (persulfato de potássio) durante os primeiros 210 minutos, quando as alimentações foram interrompidas e o processo seguiu em batelada.

Na Figura 1 apresenta-se a comparação da evolução, ao longo do tempo do processo, do diâmetro médio de partículas de polímero (medidas por espalhamento dinâmico de luz) e a concentração de partículas de polímero (calculadas a partir das medidas de conversão por gravimetria e do diâmetro das partículas). A qualidade do ajuste do modelo é muito boa para o diâmetro e aceitável para a concentração de partículas, consideradas as incertezas inerentes às medições experimentais.

A figura 2 apresenta a evolução do número médio de radicais por partícula (valor experimental obtido a partir da conversão e do diâmetro), e também a comparação da distribuição de tamanhos das partículas de polímero ao final do processo. Observa-se que o modelo captura qualitativamente a elevação do número médio de radicais ao final da fase de alimentação. A distribuição de tamanhos de partículas experimental, medida por microscopia eletrônica, apresenta-se na forma de histograma (contagem de partículas por faixas de tamanhos) enquanto que a curva do modelo é contínua e suave.

A figura 3 apresenta a comparação da evolução da concentração de monômeros nas partículas ao longo tempo medidos através de cromatografia e a fração de polímero no reator, esta obtida através de conversão por gravimetria. A qualidade ajuste foi muito aderente para a concentração de monômeros nas partículas e razoável para a evolução de fração de polímero no reator, principalmente após a interrupção da alimentação.

A modelagem matemática para este processo mostrou-se capaz de representar a evolução ao longo do tempo de diversas variáveis que podem ser utilizadas para monitorar a evolução do processo ao longo do tempo, assim como a distribuição de tamanho de partículas. 

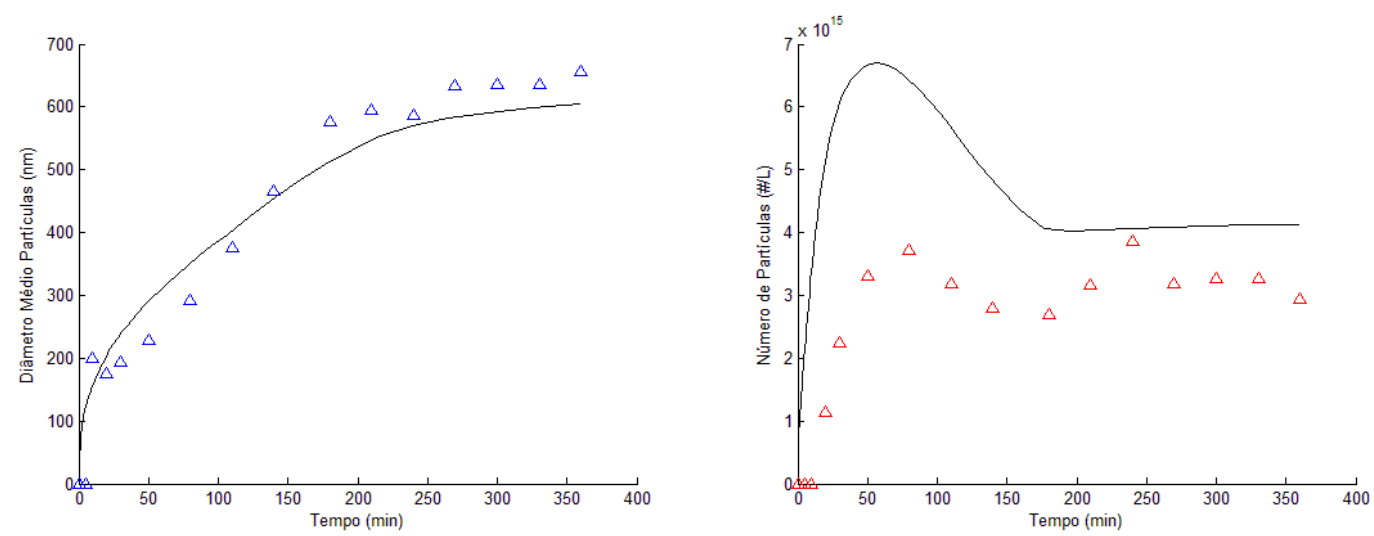

Figura 1 - Diâmetro médio das partículas e número de partículas por litro de emulsão
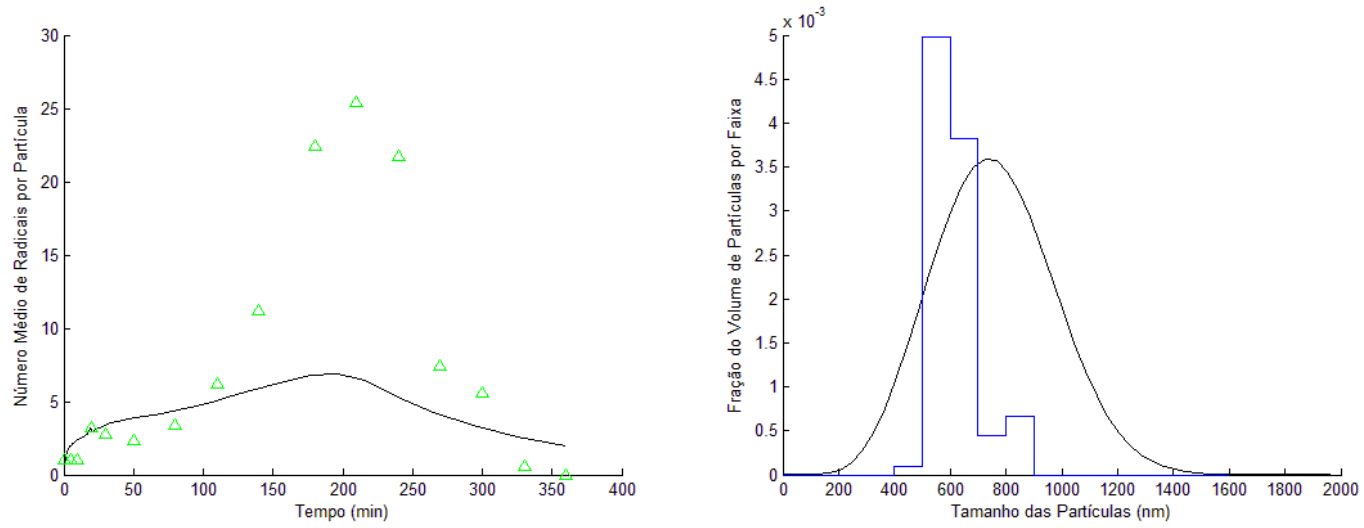

Figura 2 - Número médio de radicais por partícula e distribuição de tamanho de partículas
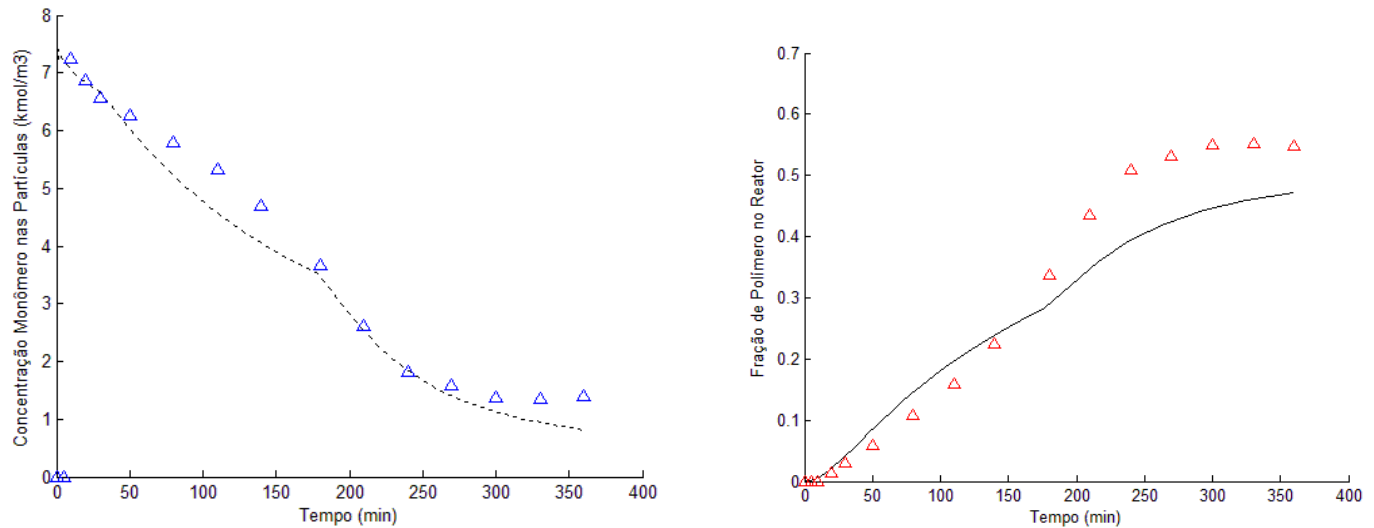

Figura 3 - Concentração de monômeros nas partículas e fração de polímero no reator. 


\section{NOMENCLATURA}

$f \quad$ Fator de eficiência do iniciador

$F(r, t) \quad$ Função de densidade probabilística

[I] Concentração de iniciador no reator

$k_{d} \quad$ Taxa de decomposição do iniciador

$k_{f m i} \quad$ Taxa de transferência para monômero

$k_{p j, a q} \quad$ Taxa de propagação na fase aquosa

$\bar{k}_{t, a q}$

$\tilde{n} \quad$ Número médio de radicais por partícula

$N_{A} \quad$ Número de Avogadro

$N_{\text {mic }} \quad$ Número de Micelas

$p_{a q, i}$

$r$

Raio da partícula

$r_{\text {nuc }} \quad$ Raio de nucleação das partículas

$r_{\max }$

$r_{\text {mic }}$

$\left[\dot{R}_{m}\right]_{a q}$

$\left[\dot{R}_{T}\right]_{a q}$

$\Re_{N u c}$

$\Re_{\text {Mic }}$

$k_{\text {emic }, i} \quad$ Constante da captura de radicais pelas micelas

$k_{p i j} \quad$ Taxa propagação entre o radical do tipo $i$ e o monômero do tipo $j$

Taxa média de terminação na fase aquosa

$\left[M_{j}\right]_{p} \quad$ Concentração do monômero $j$ nas partículas de polímero

$\left[M_{j}\right]_{a q} \quad$ Concentração do monômero $j$ na fase aquosa

$M M_{j} \quad$ Massa molecular do monômero do tipo $j$

$p_{i} \quad$ Probabilidade do radical do tipo $i$ dentro das partículas

Probabilidade do radical de tipo $i$ na fase aquosa entrar na micela

Raio máximo da partícula

$-$

$\mathrm{mol} \cdot \mathrm{m}^{-3}$

$s^{-1}$

$\mathrm{m}^{3} \cdot \mathrm{kmol}^{-1} \cdot \mathrm{s}^{-1}$

$\mathrm{kmol} \cdot \mathrm{m}^{-3} \cdot \mathrm{s}^{-1}$

$\mathrm{kmol} \cdot \mathrm{m}^{-3} \cdot \mathrm{s}^{-1}$

$\mathrm{kmol} \cdot \mathrm{m}^{-3} \cdot \mathrm{s}^{-1}$

$\mathrm{kmol} \cdot \mathrm{m}^{-3} \cdot \mathrm{s}^{-1}$

$\mathrm{kmol} \cdot \mathrm{m}^{-3}$

$\mathrm{kmol} \cdot \mathrm{m}^{-3}$

$\mathrm{kg} \cdot \mathrm{kmol}^{-1}$

$-$

$-$

$-$

$-$

$-$

$m$

$m$

$m$

$m$

Raio da Micela

Concentração de radicais na fase aquosa que podem entrar na micela

$\mathrm{kmol} \cdot \mathrm{m}^{-3}$

$\mathrm{kmol} \cdot \mathrm{m}^{-3}$

$s^{-1}$

$s^{-1}$

$s^{-1}$

$s^{-1}$

$s^{-1}$

$s^{-1}$

$S$

$m^{3}$

$m^{3}$

$\mathrm{kg} \cdot \mathrm{m}^{-3}$ 


\section{REFERÊNCIAS}

ARAÚJO, P. H. H.; GIUDICI, R. Optimization of semicontinuous emulsion polymerization reactions by IDP procedure with variable time intervals. Computers and Chemical Engineering, v.27, 2003. p.1345-1360.

ARAÚJO, P. H. H.; GIUDICI, R. Corrigendum to "Optimization of semicontinuos emuslion polymerization reactions by IDP procedure with variable time intervals". Computers and Chemical Engineering, v.28, 2004. p.575-578.

ASUA, J. M. A New model for radical desorption in emulsion polymerization. Macromolecules, v.36, 2003. p.6245-6251.

DUBÉ, M. A. et al. Mathematical modeling of multicomponet chain-growth polymerization in batch, semibatch and continous reators: A review. Industrial \& Engineering Chemistry Research, v.36, 1997. p.966-1015.

GAO, J.; PENLIDIS, A. Mathematical modeling and computer simulator/database for emulsion polymerizations. Progress in Polymer Science, v.27, 2002. p.403-535.

MARINANGELO, G.; HIROTA, W. H.; GIUDICI, R. Semi-batch emulsion copolymerization of styrene and butyl acrylate for production of high solids contents latexes: Experiments and mathematical model. Chemical Engineering Science, v.66, 2011. p.5875-5890. 